\title{
Addressing Human Error in International Space Station Flight Control Teams: Advances in Ground Training for Science Operators
}

\author{
Samantha S. Harris* \\ NASA Marshall Space Flight Center, Huntsville, AL, 35812, USA
}

\begin{abstract}
In flight control, as with any human in the loop system, operator error is an inevitable reality. On the International Space Station (ISS) where crew time and physical resources are precious and often irreplaceable, operator errors can result in significant, irreversible consequences. Flight controllers at the Payload Operations Integration Center (POIC) located at NASA's Marshall Space Flight Center (MSFC) in Huntsville, Alabama know this reality well. At the POIC, operator errors can be caused by a variety of factors, from poor hardware or software design to environmental factors such as time pressure or fatigue. The most difficult errors to address, however, are those which result from ineffective teamwork. To address these teamwork errors, trainers at the POIC have drawn best practices from high reliability industries as well as from our sister ISS control center at the Johnson Space Center (JSC) in Houston, Texas, to develop and implement a new training program focused specifically on teamwork skills. This training program, called Team Skills Training, is inspired by modern and historical training programs developed by NASA and is specifically tailored to the needs of the payload operations flight control team at the POIC. The program consists of training for both certified and trainee flight controllers, and covers team skills topics such as situational awareness, leadership, and communication skills. To maximize effectiveness, the program uses novel instructional techniques which extend beyond the classroom to encourage students to apply what they have learned to their day to day work. Minimizing operator errors in flight control is an endeavor which requires constant vigilance and continuous improvement. At the POIC, Team Skills Training is an important step in this journey.
\end{abstract}

\section{Introduction}

$\mathrm{T}$ HE International Space Station (ISS) program represents a triumph of human ingenuity. Continuously operating an international scientific laboratory in low Earth orbit (LEO) for nearly two decades has proven to be a monumental undertaking, requiring the efforts of diverse teams across the world. As with any large and complex project, the success of the ISS program is ultimately dependent on the ability of subject matter experts in a wide variety of fields to work together in cross-functional teams to achieve common goals. Teams at NASA's Payload Operations Integration Center (POIC) live this reality daily as systems experts, in many cases serving as representatives of larger specialist teams, work together to successfully execute science payload operations onboard the ISS. In a $24 \times 7$ real time operations environment, stressors such as time criticality, fatigue, and high visibility place even more pressure on teams to work together as efficiently as possible. For teams under such conditions, mastery of skills such as critical thinking, conflict management, and communication become even more critical to success, often making or breaking the ability of teams to meet objectives. Many of the human errors documented at the POIC result from breakdowns of teamwork skills in some way. Despite the recognized criticality of effective teamwork in flight control, however, teaming skills are commonly overlooked in standard training flows which tend to focus heavily on technical knowledge. In order to address human errors in team performance, it is critical to train and reinforce teaming skills beginning early in flight control training flows and continuing beyond certification.

\footnotetext{
"Payload Communications Manager, Training and Crew Operations Branch, NASA Marshall Space Flight
} Center/EO20, Huntsville, Alabama, USA. samantha.s.harris@nasa.gov 


\section{Overview of Challenges in Cross-functional Teaming}

In order to successfully execute large and complex projects like science payload operations on the ISS, experts in a wide variety of fields must work together effectively to achieve common goals. In many industries, including human space flight, this is accomplished through the use of interdisciplinary or cross-functional teams designed to leverage a variety of skill sets to create synergy through outputs that are greater than the sum of what the individual parts could produce alone. Cross-functional teams have many advantages, and are capable of tackling a wider variety of challenges and generating more diverse solution sets. The same diversity that makes interdisciplinary teams a necessary asset, however, can also lead to unique challenges which can threaten the ability of these teams to achieve goals and create high quality outputs. As cross-functional teams become the norm rather than the exception for complex human space flight projects, it is important to consider the factors which contribute to the ultimate success or failure of crossfunctional teams. Many studies have been done to this end, and some common themes emerge in the literature describing the challenges commonly experienced by cross-functional teams such as those used at the POIC.

\section{A. Cohesiveness and Trust}

Paul Santagata, Head of Industry at Google, said "There's no team without trust." While the diversity of crossfunctional teams is an important contributing factor to team creativity and success, diversity in itself is not sufficient. To succeed, the team must cooperate effectively by integrating information and perspectives in pursuit of a common goal [1]. Effective cooperation requires trust, which can be difficult to build in cross-functional teams. Multiple studies have found lower group cohesiveness in cross-functional groups as compared to single-function groups [2].This is likely due to the diversity of team members in these groups, who each bring, among other things, their own training, cultural and communication norms, functional goals, definitions of success, and reporting structures. These differences can result in stressful interpersonal relationships which hinder the development of group cohesiveness and trust $[2,3]$. Another hindrance to trust building is the inability of individuals in cross-functional groups to assess the competence level of team members from different fields of expertise [4]. In payload operations, teams are often both crossfunctional and ad hoc, or temporarily assembled for a specific purpose. On such teams, the specific makeup of specialists on the team may change from day to day, shift to shift, and sometimes even hour to hour. Payload operators must trust that their teammates (who they may like, dislike, or have never met before) are competent and reliable. This can be especially challenging in an environment when there is rarely time for the luxury of team building. Lack of trust can also hinder another critical enabling factor in cross-functional teaming, communication.

\section{B. Communication}

Trust is a key enabler of open communication on teams [5]. Cross-functional teams lacking in trust are likely to struggle to establish psychological safety, or the belief that individuals are accepted, respected, and will not be punished for mistakes. On teams with psychological safety, team members feel empowered to ask questions, share information, and make mistakes without fear of ridicule or embarrassment. In an extensive two-year study on hundreds of teams at Google named Project Aristotle, researchers discovered that psychological safety was the single most important enabling factor in team success. Furthermore, they observed that psychological safety played a critical role in empowering individuals to speak their minds, be creative, and take moderate risks. ${ }^{\dagger}$

Communication is a common challenge for most teams, and it can be especially challenging for cross-functional teams. In addition to struggles establishing trust and psychological safety, cross-functional teams may struggle with cultural differences in communication styles. Individuals are likely to implement the communication style they are accustomed to from their home organizations, and may struggle to integrate communication styles and expectations with those from groups with different cultures. Team members may be unclear on how much communication is expected, and when, with whom, and how it should be done. Even those who are willing to communicate and know how to do so may struggle to communicate the significance of their contributions to team members or leaders who do not have a strong grasp of their field of expertise. These specialists may speak their own technical languages that others do not understand, and may struggle to identify terminology that is meaningful to decision makers. Consequently, these individuals may struggle to effectively advocate for their expertise within the team. On the other end, team members may fail to give proper consideration to the contributions of others due to a lack of appreciation or understanding of other disciplines. Miscommunications such as these can be frustrating for team members and can result in conflict.

\footnotetext{
† Google Guide: Understand Team Effectiveness- https://rework.withgoogle.com/guides/understanding-team-
} effectiveness/steps/identify-dynamics-of-effective-teams/ 


\section{Conflict Management}

In addition to their challenging ad hoc nature, teams at the POIC often contend with challenges such as multiple reporting structures, limited resources, high visibility, and intense time pressure. These factors, especially when coupled with the challenges in trust building detailed above, almost inevitably result in stress and conflict. Some studies have shown conflict to be detrimental to teams, while others have shown conflict to positively influence team success and creativity. One possible explanation for this discrepancy is a distinction between the types of conflict present within the group. While research on task based conflict has shown conflicting results, relational conflict resulting from personal disagreements or negative interactions between team members is usually detrimental to team satisfaction and success [4,6]. An additional factor which seems to moderate the impact of conflict on a group is how the conflict is managed. Conflict that is managed effectively is less likely to negatively impact groups than conflict that is handled ineffectively or left unresolved [3]. On diverse teams, team members may not know enough about each other's personalities, goals, cultural norms, and fields of expertise to correctly identify true sources of the disagreement and effectively manage resulting conflicts. In such cases, it is imperative that the group leader proactively assists with managing conflict within the team.

\section{Effective Leadership}

Effective leadership is one of the most often cited enablers of cross-functional team success [7]. However, leading any team is a difficult task, and effectively leading a cross-functional team is especially so. Cross-functional team leaders must have the breadth of knowledge to see the big picture, while also understanding and appropriately valuing the contributions of team members representing a wide variety of technical disciples. They must also have the ability to manage the day to day participation and involvement of a team of diverse individuals [6]. The most successful cross-functional teams have leaders who proactively address anticipated teaming issues by establishing and enforcing shared goals and emphasizing trust building and open communication early and often. These leaders must also assume a hands-on role in conflict management by identifying and encouraging the resolution of potentially detrimental relational conflicts $[3,8]$. At the POIC, where technical experts are occasionally asked to step up as informal leaders, it is important that both formal and informal leaders are empowered and equipped with these leadership skills.

\section{Developing a Method for Training Payload Operators in Teaming Skills}

It is clear through the literature that the skills that most commonly make or break the success of crossfunctional teams are rarely technical, but are instead basic teamwork skills. Studies on errors in environments such as commercial aviation, nuclear power plants, and human space flight where humans must interact with both each other and technology have determined that the majority of accidents and incidents involve human error. These studies often cite failures in skills such as team coordination, communication, decision making, and leadership as the causes for such errors [9,10]. Despite being so often cited as sources of significant human error, these skills are often overlooked by technical trainers as basic, or assumed to be already mastered by new trainees through formal education. While most trainees do possess some level of mastery of these skills, the expectations for how they should be applied in the unique world of payload operations and real time flight control should be clarified and reinforced. Historically, cadre at the POIC have been trained on teamwork skills largely informally through feedback from training events or team interactions.

Over the years, managers and trainers at the POIC began to identify the lack of formalized team skills training for flight controllers as a risk to effective and efficient payload operations onboard the ISS. Work was done by individual trainers in isolation to fill these gaps, but integrated efforts failed to gain traction. In a paper titled Human Error and the International Space Station: Challenges and Triumphs in Science Operations presented at the 14 ${ }^{\text {th }}$ International Conference on Space Operations in Daejeon, Korea, this author identified a training program used in the commercial aviation industry called Crew Resource Management (CRM) as a promising method of training such skills [11]. CRM (initially called Cockpit Resource Management) was developed by a team at NASA in the 1970s for the commercial aviation industry to address a series of aviation disasters resulting from human errors in the cockpit. CRM has evolved a great deal over the years, but has remained focused on exposing flight crews to basic human factors knowledge and providing practical tools to help them apply those concepts operationally [10].

In late 2016, a three member task team at the POIC was assembled to explore existing training programs such as CRM and expand upon work previously done by individual trainers to develop and implement a formalized team skills training program for payload operators. Upon further research, the task team learned that CRM had already been adapted for use in human space flight by a team at the Johnson Space Center (JSC). The program, called Space Flight Resource Management (SFRM) was initially developed to help Shuttle astronauts and flight controllers manage errors during time critical events, but was later adapted for teams supporting the ISS program. A paper detailing the 
development and deployment of SFRM at JSC titled NASA's Space Flight Resource Management Program: A Successful Human Performance Error Management Program was presented at the $9^{\text {th }}$ International Conference on Space Operations in Houston, Texas [9]. The program at JSC successfully tailored the most successful elements of CRM for use in human space flight, and is still in use today. SFRM, like CRM, has evolved over the years, but the focus has remained on helping operators develop the skills necessary to manage errors and work as part of an effective team. Identifying SFRM as a promising training program, the task team at the POIC sought assistance from the SFRM training group at JSC and had the privilege of working directly with one of the original developers of SFRM who provided guidance and access to training materials.

\section{A. Tailoring SFRM to Payload Operations}

The Marshall team quickly noted that the SFRM program currently used in the Flight Operations Directorate (FOD) at JSC would provide a strong foundation for the new payload operations teaming skills program. It addressed many of the most common cross-functional team challenges, and was already working in an operational environment similar to the POIC. However, as with all training programs, it would have to be significantly tailored for successful implementation in a new organization. Heavy tailoring would have to address discrepancies in manpower, differences in organizational culture, and significant differences in the operational nature of the tasks performed by operators at the POIC compared to those in FOD. Additional tailoring would have to be done to create examples and activities representative of payload operations scenarios directly faced by students at the POIC.

- Manpower

The training organization at the POIC is much smaller than our counterpart at JSC. The SFRM training program would have to be designed in such a way that it could be developed and managed by a small group of people with limited resources.

\section{- Organizational Culture}

The organizational culture at the POIC differs from JSC in multiple key areas. Training at the POIC tends to be less rigid, with lengthy training being less common and accepted. Certified flight controllers at the POIC often consider themselves too busy to attend formalized training, especially post certification. Simultaneous efforts to adapt a different JSC training course for use at the POIC struggled due to heavy resistance to time requirement for the course (which had already been significantly truncated from the course used at JSC). A successful payload team skills training program would have to be convenient, compact, high impact, and strongly supported by management. It would also have to include components to expose both new trainees and seasoned operators to the concepts of SFRM, taking into account the unique constraints for each group. Additionally, the debrief culture at the POIC differs significantly from FOD, making it difficult to implement the student driven facilitated debriefs focused on SFRM skills which were cited as a key element of the SFRM program at JSC.

- Nature of Operational Tasks

Payload operations on the ISS are unique, and differentiated from the systems operations managed by JSC in multiple key ways. While the systems managed by JSC are relatively static, the payload complement onboard the ISS in constantly changing. Additionally, while flight controllers at JSC tend to be experts in their own systems, the team at the POIC are not experts on individual payloads. Rather, the role of flight controllers at the POIC is to represent and advocate for the payload developers and principle investigators who are the experts in their own payloads through the operations phase.

- Representation and Relevance

The existing SFRM curriculum heavily utilized JSC specific examples which would have to be tailored to ensure relevance for payload operations personnel.

\section{B. Challenges}

As the team began work tailoring SFRM concepts and training for payload operations, they also had to consider historical lessons learned and overcome challenges that arose during the development process. Significant challenges included an expansion of the target audience, resistance from employees in the target audience, and recruiting and training instructors. 


\section{- Remembering Training Lessons Learned}

The payload operations training organization was historically known for a tendency to address training holes with single, "band aid" classroom lessons. For example, when management determined that flight controllers were not displaying effective critical thinking skills on console, a single classroom lesson was created in PowerPoint and added to the training flow for new flight controllers. While this course was a concrete action to address the issue, it was a limited solution that did not effectively address the larger root cause. The organization had also experienced difficulty with bringing in outside training groups to train payload operators on soft skills. In one example, the leadership development training office at Marshall was asked to present training on stress management. While some enjoyed the class, some felt it was not tailored enough to be relevant to the unique demands and culture of payload operations. The team knew that to avoid these mistakes they would have to create a training curriculum that was comprehensive, skills based, and heavily tailored for payload operations.

\section{- Tailoring to a Diverse Audience}

The initial charter for the training development task team focused on creating a training program for real time operations flight controllers who staff consoles in the $24 \times 7$ operations center known as Payload Control Area 1 (PCA-1) shown in Figure 1. Midway through development, however, the charter expanded to include all payload support personnel in addition to real time operators. This created a new challenge to design content that was specific enough to be useful for individuals, yet general enough to be relevant to a diverse audience performing a wide variety of tasks. The development team also had to consider the requirement to train both new flight controllers and veteran flight controllers, tailoring the training to the appropriate skill level for each group.

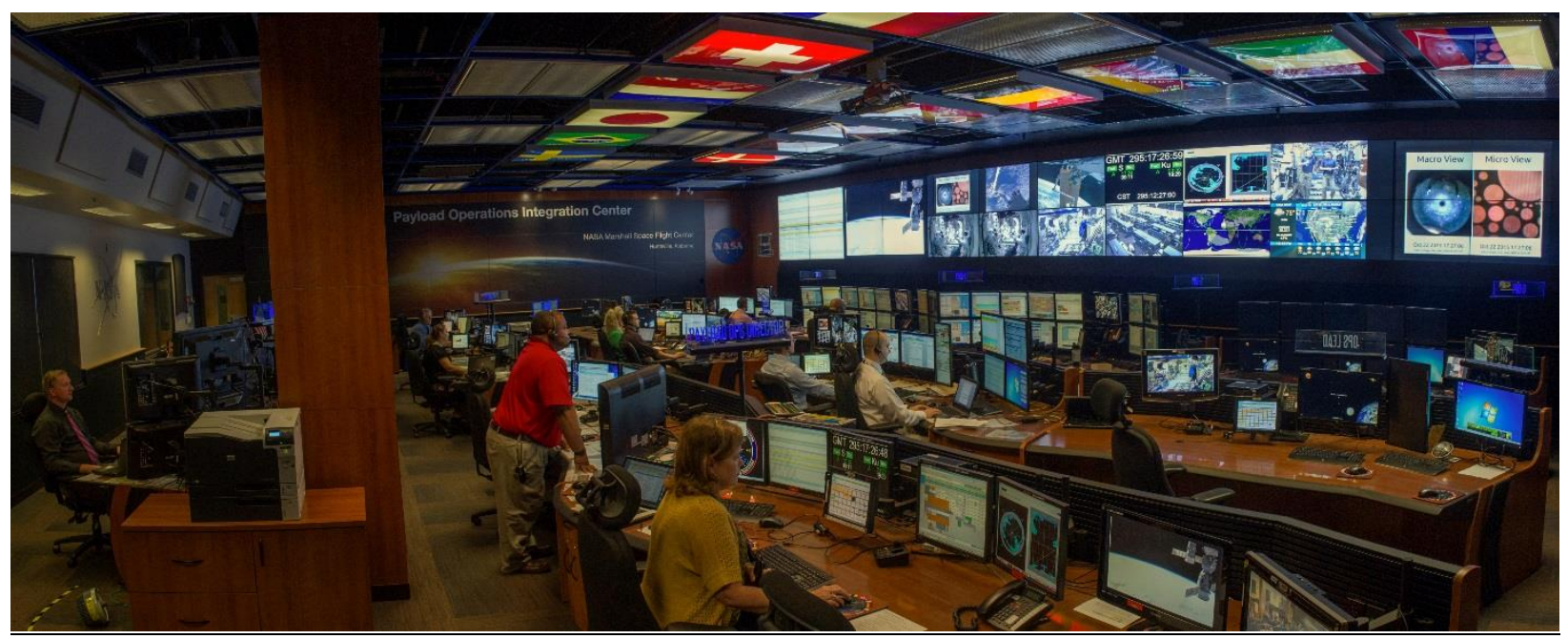

Figure 1- Payload Control Area 1

\section{- Organizational Resistance}

In addition to the challenges developing tailored content, the team faced multiple challenges in implementation. The most significant of these was obtaining buy in from the workforce. While management was supportive, the veteran technical workforce was sometimes resistant. Many veteran personnel felt they were too busy for additional training, and in some cases found the concept of mandatory team skills training unnecessary and patronizing. The technical workforce has historically been hard to sell on the value of so-called "soft skills" training, which is typically viewed as common sense, and therefore less valuable than technical training. To avoid this negative connotation, the development team intentionally chose to avoid using the term "soft skills," instead opting for the term "team skills." Because "team skills" was not a commonly recognized term, the team faced additional challenges educating both management and the work force at large on the meaning and importance of "team skills" as a proficiency.

- $\quad$ Recruiting and Training Instructors

Because the team skills development team at Marshall was so small (ultimately reducing from three part time developers to two), they knew it would be necessary to recruit additional instructors. This proved challenging for a few reasons. First, maintaining quality in the instructor pool was imperative, so not just any instructor would suffice. Successful soft skills training hinges on the skill of the instructors who must be charismatic and engaging, as well as 
proficient in a variety of instructional techniques such as facilitating student led discussions and using student questions for learning. Second, the development team wanted to use a diverse instructor pool representing a mix of fields and specialties. This created the additional logistical and political challenge of recruiting and negotiating across organizational lines.

\section{Team Skills Training Today}

Ultimately, the team settled on and developed two team skills training tracks called the Team Skills Curriculum and Team Skills Refresher Workshop. Both training tracks include classroom lessons on team dynamics, flight control criticality, communication, situational awareness, critical thinking, conflict management, giving and receiving feedback, and leadership. These topics were selected to align with common challenges in cross-functional teaming as well as the SFRM model developed at JSC. Each standalone lesson is under two hours, and is presented by a current or former flight controller who has also been trained in SFRM and instructional techniques. To ensure there are opportunities for all students to participate, the target class size is between twelve and sixteen with a cap of twenty students for all team skills courses. Lessons are presented using innovative training techniques such as student led discussions, group work, team and partner activities, and video clips from popular movies and TV shows. Students are engaged and invited to share personal experiences, examples, and applications throughout each lesson. For example, in the leadership course, students are asked to consider effective leaders they know and brainstorm a list of characteristics that make those leaders effective. They are then asked to consider a series of photos and videos of commonly known leaders such as Elon Musk and Mahatma Ghandi, and list leadership characteristics embodied by those leaders. The instructor further invites participants to consider their own leadership strengths and weaknesses, presents techniques used by veteran payload operators to exercise situational leadership on console, and ends with a team activity building rockets from Lego blocks. The feedback class includes opportunities to role play giving and receiving feedback using a feedback model presented in the class, and the situational awareness class includes an activity to build team situational awareness by using verbal communication to cooperate and place a series of photos in order.

\section{Team Skills Curriculum}

For new hires and trainees, the Team Skills Curriculum consists of a series of four, half day blocks of classroom lessons spread over time. The lessons are spread out to allow trainees time to absorb what they have learned, and to provide time for them to apply new knowledge before returning for another set of lessons. At the end of each session, trainees are asked to fill out an action plan with goals for specific skills and areas of desired improvement based on what was learned. At the start of the next block, students are asked to discuss progress made on their action plans with a partner. Some of the lessons in the curriculum go into more detail for trainees, for example the communication lesson is expanded to cover basics such as call structuring and etiquette, and more guidance may be provided by the instructor than for experienced flight controllers. Fortunately, the trainee pool is often diverse including new hires with experience in industries like the United States military, commercial aviation, healthcare, and education. This skill mix often creates robust discussions, and skilled instructors make sure to incorporate a diversity of perspectives and experiences and connect them back to payload operations. The Team Skills Curriculum was recently rolled into the mandatory new hire training flow called Payload Academy.

\section{Team Skills Refresher Workshop}

The Team Skills Refresher Workshop is targeted at veteran flight controllers, and is a more compact version of the Team Skills Curriculum. The workshop spans two, back to back eight and a half hour days. The two day workshop format was chosen to address the concerns of busy payload operators who preferred a shortened, high impact course. Lessons are largely the same as those presented to trainees, but in some cases are slightly truncated in the interest of time. Student led discussions in the workshops may be more robust and require less instructor guidance, and participants often report learning just as much from each other as they do from the formal class content. Courses are designed to provide thought provoking activities, concepts, and topics which kick off largely student led discussions. Students are invited to fill out an action plan after each lesson with goals for specific skills and areas of desired improvement based on what was learned.

\section{People Smart}

While not formally included in the team skills series of courses, an additional complementary course called People Smart was rolled out at the same time as team skills. People Smart is a two day workshop instructed by a pair of psychologists from the leadership development organization at Marshall, and focuses on the importance of leveraging ideological diversity and practical conflict resolution strategies for teams. Students are invited to first get to know 
themselves by completing a behavior assessment prior to the class. The workshop begins with a discussion of how individuals impact those around them, sometimes without realizing it, and continues into a discussion on the strengths and weaknesses of each behavioral style and the importance of leveraging the strengths of all styles on teams. Students are invited to consider how they are contributing to (or hindering) the maintenance of a psychologically safe environment on their teams. To avoid previous pitfalls associated with bringing in outside instructors who are unfamiliar with payload operations, the instructors met with representatives from the payload operations division multiple times while developing the content, and one of the primary instructors sat in on a full day simulation training event for flight controllers.

\section{Success Factors}

To date, the training group has hosted twelve Team Skills Curriculum blocks comprising approximately 70 unique trainees, ten Team Skills Refresher workshops comprising 139 students, and five People Smart workshops comprising 94 students. Anecdotal evidence and participant feedback indicate that the team skills program has been largely successful. This is particularly true for trainees, who now have teaming skills emphasized equally with technical skills beginning early in the training flow. For these operators, the difference is notable. In a small survey of 15 of the first flight controller trainees to complete the Team Skills Curriculum, 93\% of respondents reported that they had used what was learned in the program. Additionally, feedback regularly solicited from all participants in Team Skills and People Smart courses has been overwhelmingly positive. Through the experience of the team skills developers and instructors and feedback from students, common themes emerge which describe some of the reasons the program has been so successful.

\section{- Management Support}

From the beginning, the development team was fortunate to have high levels of management support for the project. The management team provided critical momentum and support throughout the development and implementation process, allowing the development team to overcome organizational inertia and hesitancy from various organizations. Additionally, management support has been instrumental in overcoming resistance from the workforce. All managers agreed to release their teams to attend the courses and, in some cases, have established requirements and due dates for their teams to complete them. Many managers have also elected to complete the courses themselves in order to better encourage their teams to make time to attend. Participant feedback indicates that even those students who are most resistant to taking the courses often learn something and leave with a more positive opinion of team skills training than they held initially. Management has also been generous in allowing the training team to recruit and utilize instructors from their organizations. The People Smart course, which receives nearly universally positive feedback and consistently holds a wait list, has an associated per student cost for the behavioral assessment which is key to the course. Management has continued to agree to fund this cost over the course of the past year since the course was implemented.

\section{- Leveraging Work Already Done}

For a small team with severely limited resources, leveraging the great work done by experts at the Johnson Space Center in Space Flight Resource Management was critical for the success of the team skills training program. While developing SFRM, the team at JSC considered a large body of academic research on best practices in CRM, and also visited and drew best practices from many high reliability organizations including nuclear power plants, commercial air carriers, and military organizations. The development team at Marshall did not have previous experience developing and facilitating team skills training, making guidance of the experts who have devoted many years of study to the practice vital. Some elements from the JSC training program, such as the Team Dynamics course and the SFRM on console aid are utilized at POIC exactly as they are at JSC. This preserved many resources, and guaranteed a high quality product.

\section{- Relevancy and Tailoring}

Many participants express surprise at how well the courses in the program are tailored to be specifically relevant to payload operations. All courses were developed by payload operators and are taught by payload operators. The curriculum is intentionally designed to address the unique challenges of payload operations, and does this well because in many cases the instructors still maintain positional certifications in a variety of technical areas.

\section{- Innovative Training Techniques}

Most training at the POIC has lagged behind evolving training techniques, and in many cases still relies on lecture style PowerPoint presentations. While this type of training can still be effective to impart technical knowledge, it is 
typically not effective to develop team skills. Participant feedback indicates that the innovative techniques employed in the team skills program, such as video clips, Lego activities and student led discussions, tend to be more enjoyable and impactful than traditional instruction methods. These techniques are used intentionally to create space for participants to reflect on the material being presented and actively consider how those skills can practically be applied in the office or on console the next day.

\section{- Train the Trainer}

In an organization with such limited resources, assembling and training what is in many cases a volunteer instructor pool was a significant challenge. Instructors needed to be trained on SFRM concepts and history, updated training techniques, and general workshop facilitation skills. To accomplish this, the team employed a train the trainer model where certified instructors are empowered to train and certify additional instructors. This allowed the team to quickly establish backup instructors, and share the workload in the early stages of implementation. It should be noted, however, that this method of instructor certification introduces risk, and can only be effective where the quality of the instructor pool is high. The team skills instructor pool is composed universally of instructors with instructional experience as well as exceptional reputations for instructional skills and technical competency.

- Openness to Feedback

Possibly the greatest strength of the team skills training program is the openness of the development and instructional teams to feedback. Participants are polled for feedback after all courses, and asked what they enjoyed and what they would improve about the course. All instructors are provided with the feedback, and discuss as a group what changes should be made between courses. It is rare for identical courses to be presented because improvements are constantly being considered and implemented. When developing new training in a non-technical competency such as team skills, especially if the training developers don't have direct experience creating and facilitating such training, continuous improvement and responsiveness to student and expert feedback are critical. Instructors must consistently evaluate what works and what doesn't, and make appropriate changes.

\section{Forward Work}

While trainers at the POIC have accomplished a great deal by developing and implementing a basic SFRM based team skills training program, there is still work to be done. In the spirit of continuous improvement, the team is always considering what can be improved using available resources. Through feedback, experience, and guidance from trainers at JSC, the team has identified short term areas of improvement designed to further increase the impact of the training program. These improvements include further tailoring of instructional material, the introduction of low fidelity simulations, increasing the amount of reinforcement trainees and veteran controllers receive on team skills proficiency, and developing additional measures of effectiveness.

- Further Tailor Content for Payload Specific Scenarios

While the team skills courses are heavily tailored for payload operations, there is still room for improvement. Feedback has indicated that students would like to see more specific payloads focused videos, examples and activities. The development team would like to add more robust content using real payload operations scenarios and audio clips from training and real time events. For example, audio clips of effective communication or a mistake made by the real time team can be used to initiate discussion. Instructors are also striving to continue to include more personal "war stories" and experiences from their own work as flight controllers.

\section{- Low Fidelity Simulations}

The JSC SFRM program includes the use of low fidelity table top simulations based on a moon expedition scenario. The simulations require limited training resources and provide students with an important opportunity to practice teaming skills learned in the classroom in a non-technical, lower pressure environment than typical training simulations. In the simulations, teams of students man four lunar rovers and one mission control center. Students must work effectively and efficiently as a team to manage limited time and resources in order to successfully complete two primary objectives defined as mission safety and full mission success. The simulations are followed by debriefs, in which students are encouraged to practice identifying effective and non-effective examples of team skills. In 2017, a trainer from JSC traveled to Marshall to train payload trainers in how to successfully run moon base simulations, and roll out at the POIC is slated for mid-2018. 
- Increase Reinforcement throughout the Flight Control Experience

Team skills training is a still a relatively new concept at the POIC for veteran operators as well as for trainees. Many veteran operators have not yet received training in SFRM or teaming skills, and therefore do not consistently provide effective feedback or mentorship on teaming skills to trainees during events such as on the job training and simulations. Once the full flight control complement has been exposed to team skills concepts, training can be created to encourage more robust team skills feedback and reinforcement throughout all stages of the feedback and training flows. Eventually, all payload operators will be required to go through team skills training and measures are being considered to hold veteran operators accountable for their mastery of team skills through proficiency training efforts and evaluations.

- Expand Effectiveness Measures

It is often difficult to gauge the effectiveness of soft skills training such as the team skills program. To date, measures of effectiveness have largely been based on anecdotal evidence and participant feedback after training events. While this is useful information and has created many opportunities for improvement, it is not enough to prove that the training is creating measurable change. The team would like to introduce additional effectiveness measures to determine whether team skills training is in fact improving human error management and resulting in more effective teamwork at the POIC. One way this could be accomplished is through a more robust and reliable error reporting system, which is already being considered for implementation.

\section{Conclusion}

Over the past two years, the training flow for flight controllers at the Payload Operations Integration Center for the International Space Station has gone through a significant evolution as team skills training has been formalized and elevated to equal emphasis and importance with technical skills. Courses in teaming skills such as communication, situational awareness, conflict management, and leadership are now standard practice for all trainee and veteran flight controllers, and are presented using innovative training techniques shown to increase the effectiveness of soft skills training such as the team skills program. This is a momentous step, as studies continue to show that human error accounts for the majority of mistakes and accidents in human space flight and similar fields. A review of errors made by operators at the POIC shows a similar trend in team skills errors that have historically been difficult to address. This is not unusual, as cross-functional teams in industries from spaceflight and aviation to oil rigs and healthcare have struggled with similar challenges in cooperation, communication, conflict management, and leadership. The newly implemented team skills training program at the POIC draws from best practices in Crew Resource Management and Space Flight Resource Management to address these issues and more. As new payloads continue to join the complement onboard the ISS and crew time devoted to science increases, payload operators are under more pressure than ever before to perform as an efficient, effective, and innovative team. While human errors will always be a reality in human space flight, a well-designed and implemented team skills training program has the potential to decrease errors in payload operations in the same way CRM decreased errors in commercial aviation and SFRM decreased errors in human space flight at Mission Control Houston. The true value of such training programs, however, lies not in classroom lesson or even practice scenarios, but in the recognition of teaming skills as critical to successful error management and team performance. While not a panacea, the newly implemented team skills training program for flight controllers at the POIC is an important step in the right direction.

\section{Acknowledgments}

The author would like to recognize and thank Ann Bathew, Lisa Mavrotheris, and Craig Cruzen for their efforts as part of the team skills training development team. She would also like to thank the management team, including Jason Norwood and Carmen Price, for supporting the development and implementation of this training. Finally, the author would like to thank Therese Huning at Johnson Space Center for her guidance and support during the team skills training development effort. As one of the original developers and instructors of Space Flight Resource Management, her expertise was invaluable.

\section{References}

[1] Parker, Glenn M. Cross-functional Teams: Working with Allies, Enemies, and Other Strangers, Jossey-Bass, San Francisco, 1994, Chaps. 1, 3, 4.

[2] Keller, R. T., "Cross-Functional Project Groups in Research and New Product Development: Diversity, Communications, Job Stress, and Outcomes,"Academy of Management Journal, Vol. 44, No. 3, 2001, pp. 547-555. doi: 10.2307/3069369 
[3] Webber, S.S., "Leadership and Trust Facilitating Cross-Functional Team Success," Journal of Management Development, Vol. 21, No. 3, pp.201-214.

doi: $10.1108 / 02621710210420273$

[4] Yong, K., Sauer, S.J., and Elizabeth A. Mannix, "Conflict and Creativity in Interdisciplinary Teams," Small Group Research, Vol. 45, No. 3, May 2014, pp. 266 - 289. doi: $10.1177 / 1046496414530789$

[5] Denison, D., Hart, S., and Kahn, J., "From Chimneys to Cross-Functional Teams: Developing and Validating a Diagnostic Model," The Academy of Management Journal, Vol. 39, No. 4, August 1996, pp. 1005-1023. doi: $10.2307 / 256721$

[6] De Dreu, C. and Weingart, L., "Task versus Relationship Conflict, Team Performance, and Team Member Satisfaction: A Meta-Analysis," Journal of Applied Psychology, Vol. 88, No. 4, 2003, pp. 741-749. doi: 10.1037/0021-9010.88.4.741

[7] Nancarrow, S. A., Booth, A., Ariss, S., Smith, T., Enderby, P., and Roots, A., "Ten Principles of Good Interdisciplinary Team Work," Human Resources for Health, Vol. 11, No. 19, May 2013. doi: 10.1186/1478-4491-11-19

[8] McDonough III, E.F., "Investigation of Factors Contributing to the Success of Cross-Functional Teams," Journal of Product Innovation Management, Vol. 17, No. 3, May 2000, pp. 221-235. doi: 10.1016/S0737-6782(00)00041-2

[9] Derone Jenkins-Todd, Ph.D., Ronda Kempton, Brad Miller, David G. Rogers, John Bauer, Steven F. Dillon, Therese M. Hunting, and Michael R. Sterling. "NASA's Space Flight Resource Management Program: A Successful Human Performance Error Management Program", SpaceOps 2002 Conference, SpaceOps Conferences. doi: $10.2514 / 6.2002-\mathrm{T} 4-12$

[10] Helmreich, R. L., and Foushee, C.H., Crew Resource Management, 2nd ed., edited by B.G. Kanki, R.L. Helmreich and J. Anca, Academic Press, San Diego, 2010, Chap. 1. doi: 10.1016/B978-0-12-374946-8.10026-3

[11] Samantha Harris and Beau Simpson. "Human Error and the International Space Station: Challenges and Triumphs in Science Operations", SpaceOps 2016 Conference, SpaceOps Conferences, (AIAA 2016-2406). doi: $10.2514 / 6.2016-2406$ 